\title{
Cryptotanshinone suppresses the proliferation and induces the apoptosis of pancreatic cancer cells via the STAT3 signaling pathway
}

\author{
YUQING GE $^{1}$, BO YANG ${ }^{2}$, ZHE CHEN ${ }^{1}$ and RUBIN CHENG $^{2}$ \\ ${ }^{1}$ National Clinical Research Base of Traditional Chinese Medicine, The First Affiliated Hospital, \\ Zhejiang Chinese Medical University; ${ }^{2}$ College of Pharmaceutical Science, \\ Zhejiang Chinese Medical University, Hangzhou, Zhejiang 310053, P.R. China
}

Received December 9, 2014; Accepted August 27, 2015

DOI: $10.3892 / \mathrm{mmr} .2015 .4379$

\begin{abstract}
Pancreatic cancer remains a challenging disease worldwide. Cryptotanshinone (CPT) is one of the active constituents of Salvia miltiorrhiza Bunge and exhibits significant antitumor activities in several human cancer cells. However, the efficacy and molecular mechanism of CPT in pancreatic cancer remains to be elucidated. In the present study, the effect of CPT on the proliferation, apoptosis and cell cycle of human pancreatic cancer cell BxPC-3 cells was evaluated. The results demonstrated that CPT inhibited proliferation of the BxPC-3 cells in a concentration-dependent manner, and significantly induced cell apoptosis and cell cycle arrest. The protein levels of cleaved caspase-3, caspase- 9 and poly ADP ribose polymerase were upregulated, while the levels of c-myc, survivin and cyclin D1 were downregulated following treatment with CPT. In addition, CPT decreased the activities of signal transducer and activator of transcription 3 (STAT3) and several upstream regulatory signaling pathways after $24 \mathrm{~h}$. However, CPT only inhibited the phosphorylation of STAT3 Tyr705 within $30 \mathrm{~min}$, without marked effects on the phosphorylation of the other proteins. These results suggested that the inhibition of STAT3 activity by CPT was directly and independent of the upstream regulators in human pancreatic cancer. The present study demonstrated that CPT exerts anticancer effects by inducing apoptosis and cell cycle arrest via inhibition of the STAT3 signaling pathway in human BxPC-3 cells.
\end{abstract}

Correspondence to: Dr Rubin Cheng, College of Pharmaceutical Science, Zhejiang Chinese Medical University, 548 Binwen Road, Hangzhou, Zhejiang 310053, P.R. China

E-mail: biothcheng@hotmail.com

Key words: pancreatic cancer, cryptotanshinone, cell apoptosis, signal transducer and activator of transcription 3 signaling pathway

\section{Introduction}

Pancreatic cancer is the fourth leading cause of cancer-associated mortality, and is amongst the most life-threatening types of human cancer. Its 5 -year-survival rate is $<5 \%$ due to its aggressive growth, metastasis and resistance to the majority of chemotherapies (1). Systemic chemotherapy remains reliant on a few drugs and has not significantly increased overall patient survival rates (2). Therefore, more effective therapeutic approaches are necessary for improving the outcome of pancreatic cancer treatment.

The signal transducer and activator of transcription 3 (STAT3) transcription factor is constitutively activated in several human cancer cells, and aberrant STAT3 signaling is implicated as an important process in malignant progression (3). When STAT3 is activated upon phosphorylation of the tyrosine 705 residue, monomeric STAT3 forms dimers through their SH2 domains and translocates from the cytoplasm into the nucleus. Once in the nucleus, STAT3 transcriptionally regulates the expression of its target genes, including cyclin D1 and survivin (4). The activation of the STAT3 pathway can stimulate cell proliferation, promote angiogenesis, mediate immune evasion and confer increased resistance to apoptosis in cancer cells $(5,6)$. STAT3 is found to be constitutively activated in pancreatic ductal adenocarcinoma (PDAC) through the phosphorylation of Tyr705, which has been reported in human tumor specimens and in various PDAC cell lines. Aberrant activation of STAT3 in the pancreas accelerates pancreatic intraepithelial neoplasia progression and PDAC development (7), and activated STAT3 signaling prevents cell apoptosis and confers resistance to chemotherapeutic agents in pancreatic carcinogenesis (8). Furthermore, STAT3 knockdown reduces pancreatic cancer cell invasiveness and expression of matrix metalloproteinase-7 in nude mice (9). Inhibition of STAT3 activity by sorafenib also enhances TNF-related apoptosis-inducing ligand-mediated apoptosis in human pancreatic cancer cells (10). Thus, STAT3 signaling has been considered as an attractive chemotherapeutic target for the treatment of pancreatic cancer.

Cryptotanshinone (CPT) is one of the major tanshinones isolated from Salvia miltiorrhiza Bunge, a well-known 
Chinese herb, and is widely used in traditional and modern medicine (11). Extensive investigations have indicated that CPT may inhibit the proliferation of cancer cells in vitro and reduce the growth and metastases of tumors in vivo (11-13). CPT inhibits the function of STAT3 though inhibiting dimerization in DU145 prostate cancer cells (14). In addition, CPT significantly decreases the activity of the STAT3 signaling pathway in human glioma and chronic myeloid leukemia cells, indicating that CPT is a potent STAT3 inhibitor $(15,16)$. However, the effect and mechanism of CPT on human pancreatic cancer remains to be elucidated. In the present study, the effects of $\mathrm{CPT}$ on the proliferation and apoptosis of BXPC-3 human pancreatic cancer cells were evaluated, and the underlying molecular mechanism was investigated. Elucidating of the effects of CPT on the apoptosis of BXPC-3 pancreatic cancer cells and on the STAT3 pathway aimed to provide evidence for CPT as a potential therapeutic agent for pancreatic cancer.

\section{Materials and methods}

Cell culture. The human BxPC-3 pancreatic cancer cell line was obtained from the Type Culture Collection of the Chinese Academy of Sciences (Shanghai, China). The cells were grown in RPMI-1640 containing $10 \%$ fetal bovine serum, $100 \mu \mathrm{g} / \mathrm{ml}$ streptomycin and $100 \mathrm{U} / \mathrm{ml}$ of penicillin at $37^{\circ} \mathrm{C}$ in a humidified atmosphere of $5 \% \mathrm{CO}_{2}$.

Drugs and reagents. CPT (Fig. 1A) was purchased from the National Institutes for Food and Drug Control (Beijing, China), and was diluted using DMSO in a stock concentration of $20 \mathrm{mM}$ at $-20^{\circ} \mathrm{C}$. The methyl thiazolyl tetrazolium (MTT) and DMSO were obtained from Sigma-Aldrich (St. Louis, MO, USA). Rabbit anti-human cleaved caspase-3 (Asp175/5A1E) monoclonal antibody (cat. no. 9664), rabbit anti-human cleaved caspase-9 (Asp330) polyclonal antibody (cat. no. 9501), rabbit-anti human cleaved poly ADP ribose polymerase (PARP; Asp214/D64E10) monoclonal antibody (cat. no. 5625), rabbit anti-human phosphorylated (p-)STAT3 (Tyr705) polyclonal antibody (cat. no. 9131), rabbit anti-human p-Akt (Ser473) polyclonal antibody (cat. no. 9271), rabbit anti-human p-p44/42 extracellular signal-regulated kinase (ERK)1/2; Thr202/Tyr204) polyclonal antibody (cat. no. 9101), rabbit anti-human p-mammalian target of rapamycin (mTOR; Ser2448) polyclonal antibody (cat. no. 2971) and rabbit anti-human $\beta$-actin polyclonal antibody (cat. no. 4967) were purchased from Cell Signaling Technology, Inc. (Danvers, MA, USA). Rabbit anti-human survivin (FL-142) polyclonal antibody (cat. no. sc-10811) and rabbit anti-human Bcl-2 polyclonal antibody (cat. no. sc-783) were purchased from Santa Cruz Biotechnology, Inc. (Dallas, TX, USA). Mouse anti-human c-Myc monoclonal antibody (cat. no. 551101) and mouse anti-human Cyclin D1 monoclonal antibody (cat. no. 556470) were purchased from BD Biosciences, (Franklin Lakes, NJ, USA). Rabbit anti-human phospho-Jak2 (Y1007/Y1008) monoclonal antibody (cat. no. ab32101) was purchased from Abcam (Cambridge, MA, USA). Secondary antibodies of goat anti-mouse IgG-horesradish peroxidase (HRP; cat. no. sc-2005) and goat anti-Rabbit IgG-HRP (cat. no. sc-2004) were obtained from Santa Cruz Biotechnology, Inc.). The cell cycle detection kit (cat. no. 558662) and fluorescein isothiocyanate-conjugated
Annexin V Apoptosis Detection kit (cat. no. 556547) were purchased from BD Biosciences.

MTT assay. Exponentially growing BxPC-3 cells were seeded into 96-well plates, at a density of $1 \times 10^{4}$ cells per well, for viability measurements, and were incubated for $12 \mathrm{~h}$ at $37^{\circ} \mathrm{C}$. To examine the proliferation inhibitory effect, different concentrations of CPT $(0,5,10,20,30$ and $40 \mu \mathrm{M})$ were added to the wells, with a total volume of $200 \mu \mathrm{l}$ in RPMI 1640, and incubated for 24 or $48 \mathrm{~h}$ at $37^{\circ} \mathrm{C}$. Cells treated with medium served as a control. Finally, $20 \mu \mathrm{l}$ MTT was added into each well and the absorbance at $570 \mathrm{~nm}$ was measured using a microplate spectrophotometer (SpectraMax; Molecular Devices, Sunnyvale, CA, USA). The viabilities of the cells in each well were indicated by the measured values. Treatment effects were determined as the percentage of viable cells, compared with the untreated control cells. Three independent sets of experiments were performed.

Apoptosis assay. To determine effects of CPT on apoptosis, $1 \times 10^{6} \mathrm{BxPC}-3$ cells were plated in 6 -well plates with medium for $12 \mathrm{~h}$. The cells were then treated for a further $24 \mathrm{~h}$ with $0,10,20,30$ or $40 \mu \mathrm{M}$ CPT. Subsequently, the floating and adherent cells were collected together for analysis. The cells were washed twice with cold phosphate-buffered saline (PBS), and resuspended in Annexin-Binding buffer (1X; BD Biosciences) at a concentration of $1 \times 10^{6}$ cells per $\mathrm{ml}$. Subsequently, $100 \mu \mathrm{l}$ of the cell solution was stained with $5 \mu \mathrm{l}$ Annexin $\mathrm{V}$ and $5 \mu \mathrm{l}$ propidium iodide $(\mathrm{PI})$ at room temperature for $20 \mathrm{~min}$ in the dark. The data were collected and analyzed using a FACScalibur flow cytometer with CellQuest Software (Version 5.1.1; BD Biosciences). Three independent sets of experiments were performed.

DAPI staining assay. The BxPC-3 cells were seeded within chamber dishes and treated with or without $30 \mu \mathrm{M} \mathrm{CPT}$ for $24 \mathrm{~h}$ at $37^{\circ} \mathrm{C}$. Following treatment, the cells were washed with twice ice-cold PBS and then fixed with ethanol for $30 \mathrm{~min}$ at $-20^{\circ} \mathrm{C}$. The fixed cells were then washed with PBS and stained with $10 \mu \mathrm{g} / \mathrm{ml}$ DAPI (Nanjing KeyGen Biotech Co., Ltd., Nanjing, China) for $15 \mathrm{~min}$ at room temperature. Following washing three times with PBS, the cells were observed under a fluorescence microscope (IX2-ILL 100; Olympus Corporation, Tokyo, Japan).

Cell cycle analysis. The BxPC-3 cells were seeded into 6-well plates and were treated with series of concentrations of CPT $(0,10,20,30$ and $40 \mu \mathrm{M})$ for $24 \mathrm{~h}$ at $37^{\circ} \mathrm{C}$. The cells were harvested and fixed in $70 \%$ ethanol and stored at $4^{\circ} \mathrm{C}$ overnight. Subsequently, the cell pellets were suspended in $0.5 \mathrm{ml}$ of PI solution containing $5 \mu \mathrm{g} / \mathrm{ml}$ RNase (Sigma-Aldrich) and $20 \mu \mathrm{g} / \mathrm{ml}$ PI. Following incubation in the dark for $30 \mathrm{~min}$ at $37^{\circ} \mathrm{C}$, the percentages of cells within each stage of the cell cycle (G0-G1, S or G2-M) were determined using flow cytometry (FACSCalibur, BD Biosciences). Cells treated with vehicle alone (100\% ethanol) were used as a control. At least 10,000 events were counted for each sample.

Western blot analysis. Total proteins were extracted using Radioimmunoprecipitation Assay lysis buffer (Beyotime 
A

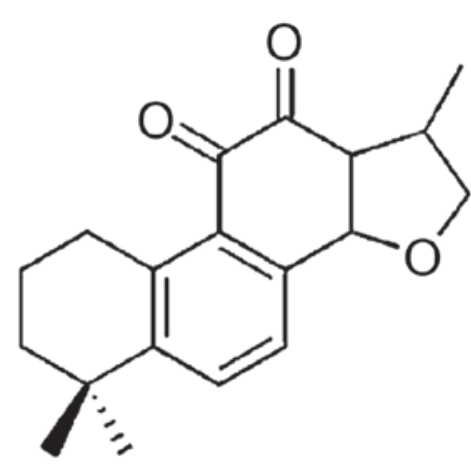

Cryptotanshinone (CPT)

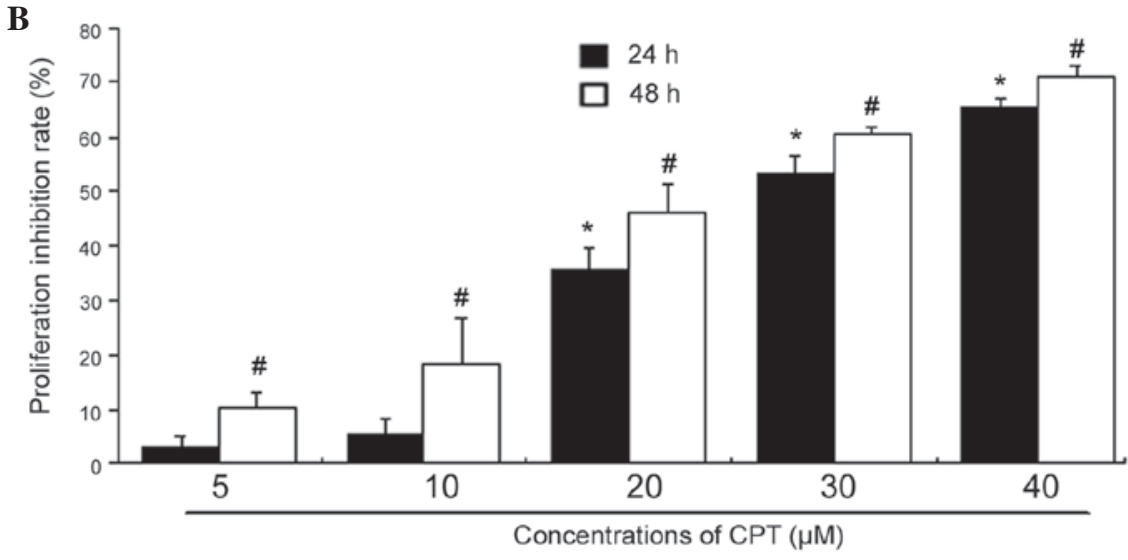

Figure 1. Effect of CPT on the proliferation of BxPC-3 human pancreatic cancer cell cells. (A) Chemical structure of CPT. (B) Cells were treated with the indicated concentrations of CPT for 24 or $48 \mathrm{~h}$, and the inhibition of cell proliferation was determined using a methyl thiazolyl tetrazolium assay. Data are expressed as the mean \pm standard deviation. ${ }^{*} \mathrm{P}<0.05$, vs. control group after $24 \mathrm{~h} \mathrm{CPT} \mathrm{treatment;}{ }^{*} \mathrm{P}<0.05$, vs. control group after $48 \mathrm{~h} \mathrm{CPT}$ treatment. $\mathrm{CPT}$, cryptotanshinone.

Institute of Biotechnology, Haimen, China), according to the manufacturer's instructions. The protein contents were determined using a Bradford assay kit (Nanjing KeyGen Biotech Co., Ltd.). Subsequently, $\sim 24 \mu \mathrm{g}$ total protein was resolved on $10 \%$ SDS-polyacrylamide gels on a Minigel apparatus (Bio-Rad Laboratories, Inc., Hercules, CA, USA), and were then transferred onto a polyvinylidene fluoride membrane (EMD Millipore, Billerica, MA, USA) by semidry transfer cell. The membranes were washed three times and blocked with Tris-buffered saline with Tween 20 (0.5\% Tween) containing $5 \%$ nonfat milk for $60 \mathrm{~min}$, followed by incubation of the membrane with the appropriate primary antibodies $(1: 1,000)$ at $4^{\circ} \mathrm{C}$ overnight, the membranes were washed in TBST and were subsequently incubated with horseradish peroxidase-conjugated secondary antibodies $(1: 2,000)$ for $2 \mathrm{~h}$ at room temperature, immunoreactive bands were developed using enhanced chemiluminescence (WBKLS0100; EMD Millipore). $\beta$-actin was stained as a loading control. The density of the blot was further analyzed by densitometry using a Gel-Pro Analyzer (Universal Hood II; Bio-Rad Laboratories, Inc.). All western blot experiments were performed at least three times. The blots depicted in the figures are a single blot, representative of three independent experiments, with density data collected from the average values of the three.

Statistical analysis. All assays in the present study were repeated independently three times. For analysis of experimental data, comparison of categorical data was performed using Student's t-test. Statistical analysis was performed using SPSS version 17.0 (SPSS, Inc., Chicago, IL, USA). Data are presented as the mean \pm standard deviation. $\mathrm{P}<0.05$ was considered to indicate a statistically significant difference.

\section{Results}

CPT has an antiproliferative effect on BXPC-3 cells. The effect of CPT on the proliferation of the BxPC-3 human pancreatic adenocarcinoma cell line was determined using an MTT assay. As shown in Fig. 1B, CPT inhibited the proliferation of BxPC-3 cells in a dose- and time-dependent manner.
Following culture with 20 or $30 \mu \mathrm{M} \mathrm{CPT}$ for $24 \mathrm{~h}$, the percentages of cell viability, relative to the control were 74 and $46 \%$, respectively. Following CPT treatment for 24 and $48 \mathrm{~h}$, the half maximal inhibitory concentration values for the BxPC-3 cells were 29.4 and $22.8 \mu \mathrm{M}$, respectively. These results indicated that CPT may function as a tumor suppressor in human pancreatic cancer.

CPT induces apoptosis and cell cycle arrest in BxPC-3 cells. To determine whether the CPT-mediated growth inhibition in the $\mathrm{BxPC}-3$ is associated with apoptosis, the percentage of apoptotic cells were determined using flow cytometric analysis with combined Annexin-V/PI staining following treatment with CPT for $24 \mathrm{~h}$. Compared with the control group, the apoptotic cell fractions were significantly increased following treatment with CPT. In the untreated control group, the percentage of apoptotic cell fractions was only $9.9 \%$. The addition of 20 and $30 \mu \mathrm{M}$ CPT to the BxPC-3 cells resulted in apoptotic cell fractions of 25.3 and $56.7 \%$, which were 2.56- and 5.73-fold higher than those of the control group, respectively (Fig. 2A). The observations performed under a light microscopic also suggested the proliferation inhibiting and apoptosis inducting effects of CPT (Fig. 2B). To further confirm the induction of apoptosis by CPT, the cells were stained with DAPI and observed under fluorescence microscopy. As shown in Fig. 2B, a normal homogeneous distribution of chromatin was observed in the untreated control cells, whereas the cells treated with CPT exhibited morphological features of apoptotic cells, including chromatin condensation and marginalization or DNA fragmentation.

To determine the effect of CPT on the cell cycle, the BxPC-3 cells were treated with increasing concentrations of CPT for $24 \mathrm{~h}$, stained with PI and assayed using flow cytometry (Fig. 2C). The results indicated that CPT treatment arrested the BxPC-3 cells in the G1-G0 phase of the cell cycle, in a dose-dependent manner. As shown in Fig. 2C, the BxPC-3 cells without CPT exposure presented with $42.39 \%$ of the population in the G1-G0 phase. However, the G1-G0 phase fraction increased to 51.22 and $75.49 \%$, following treatment with 30 and $40 \mu \mathrm{M}$ CPT, respectively. This increase in the 
A
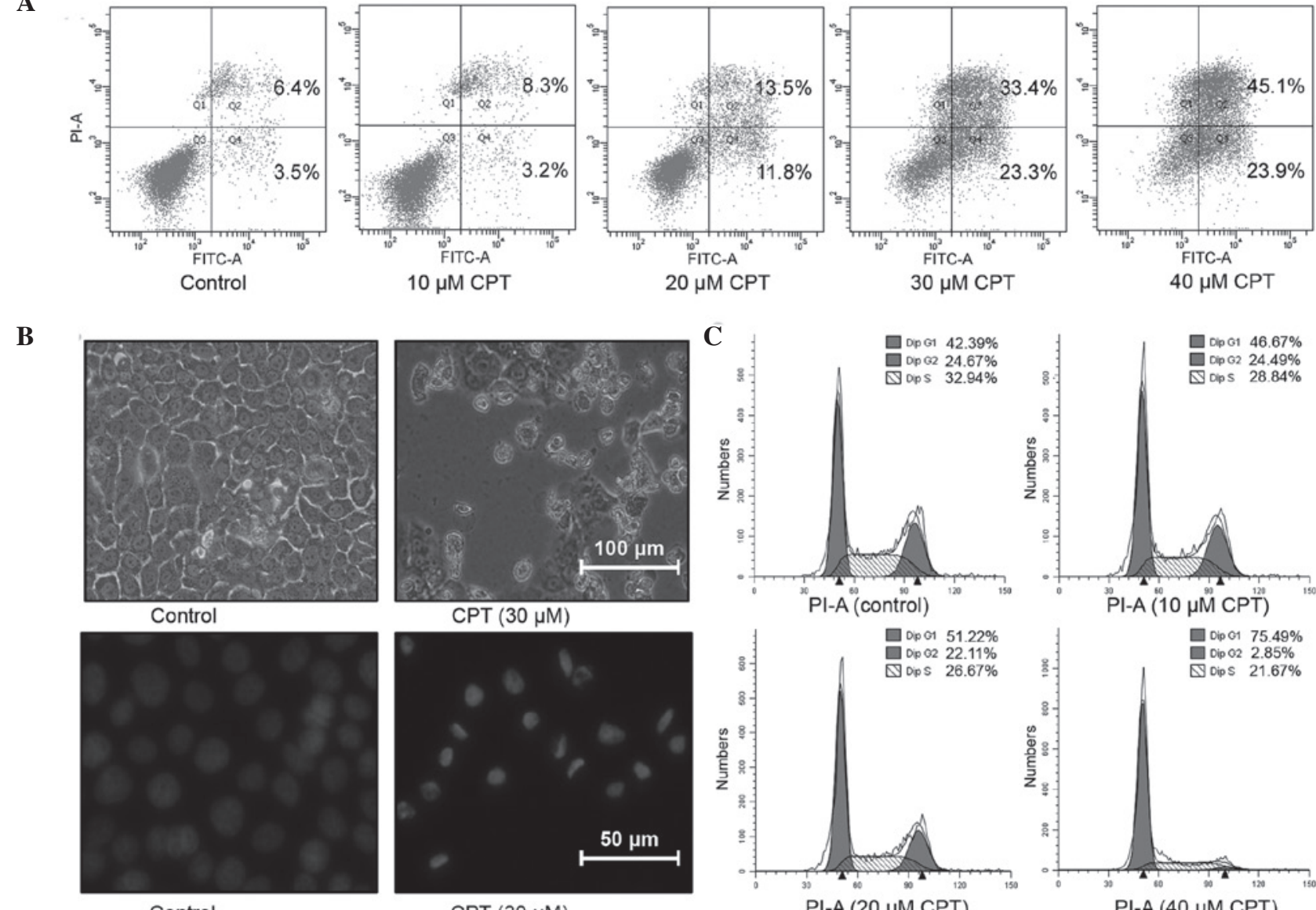

Control

$\mathrm{CPT}(30 \mu \mathrm{M})$

PI-A $(20 \mu \mathrm{M}$ CPT $)$

$\mathrm{PI}-\mathrm{A}(40 \mu \mathrm{M}$ CPT$)$

Figure 2. Effect of CPT on the apoptosis and cell cycle of BxPC-3 cells. Cells were treated with different concentrations of CPT for $24 \mathrm{~h}$. The cell apoptosis was analyze by (A) Annexin V binding and (B) DAPI staining. For each representative dot-plot, the percentages of cells in early and late apoptosis are indicated in the relative quadrant. (C) Cell cycle progression in the BxPC-3 cells was assessed using flow cytometry. Cells were fixed, stained and analyzed for DNA content. The distribution and percentages of cells in the G1/G0, S and G2/M phases of the cell cycle are indicated. CPT, cryptotanshinone; PI, propidium iodide; FITC, fluorescein isothiocyanate; A, Annexin.

G1-G0 cell population was accompanied by a concomitant decrease in the number of cells in the S-phase and G2-M phase of the cell cycle (Fig. 2C). These results demonstrated that the inhibition of cell proliferation in BxPC-3 cells by CPT was associated with the induction of apoptosis and arrest of the cell cycle in BxPC-3 cells.

$C P T$ regulates the expression levels of apoptosis- and cell cycle-associated proteins in BXPC-3 cells. To further elucidate the molecular mechanisms underlying the effects of CPT in human pancreatic cancer, the preset study investigated the expression levels of protein markers, which are associated with apoptosis and the cell cycle, following CPT treatment. As shown in Fig. 3, CPT treatment markedly increased the activities of pro-apoptotic proteins in BxPC-3 cells, including PARP, caspase- 3 and caspase-9. For example, following treatment with $30 \mu \mathrm{M} \mathrm{CPT}$ for $24 \mathrm{~h}$, the cleavage of PARP and caspase- 3 were 3.6 and 2.9-folds higher, respectively, compared with the levels in the control group (Fig. 3). Although CPT did not inhibit the protein expression of anti-apoptotic bcl-2, it significantly decreased the expression levels of c-myc and survivin, which are two important regulators for cell growth and apoptosis in cancer cells $(17,18)$. Following treatment with $30 \mu \mathrm{M}$ CPT for $24 \mathrm{~h}$, the expression levels of c-myc and survivin reduced by 35 and $76 \%$, respectively. In addition, the expression of cyclin D1 was also significantly downregulated by CPT in a dose-dependent manner, suggesting that CPT arrested cells at the G1-G0 phase by inhibiting the expression of cyclin D1 in human pancreatic cancer. These results further confirmed the apoptosis induction and cell cycle arrest induced by CPT in the human pancreatic cancer.

CPT inhibits the STAT3 signaling pathway in BXPC-3 cells. As the pivotal role of STAT3 signaling pathway is in tumor growth and apoptosis, the present study examined whether CPT had an effect on the constitutively active STAT3 in BxPC-3 cells. As shown in Fig. 4A, CPT inhibited the phosphorylation of STAT3 at Tyr705 in a dose-dependent manner, without marked effects on the total protein level of STAT3. Following exposure to $20 \mu \mathrm{M} \mathrm{CPT}$ for $24 \mathrm{~h}$, the expression of p-STAT3 was almost undetectable in the BxPC-3 cells. To clarify the mechanism underlying the inhibition of STAT3 phosphorylation by CPT, the activities of STAT3 upstream regulators were also determined following CPT exposure (Fig. 4A). The results of the western blot analysis indicated that CPT decreased the phosphorylation of the JAK2, Akt, mTOR and ERK proteins only when the concentrations of CPT increased to $20 \mu \mathrm{M}$. The phosphorylation of STAT3 was inhibited $\sim 70 \%$ 

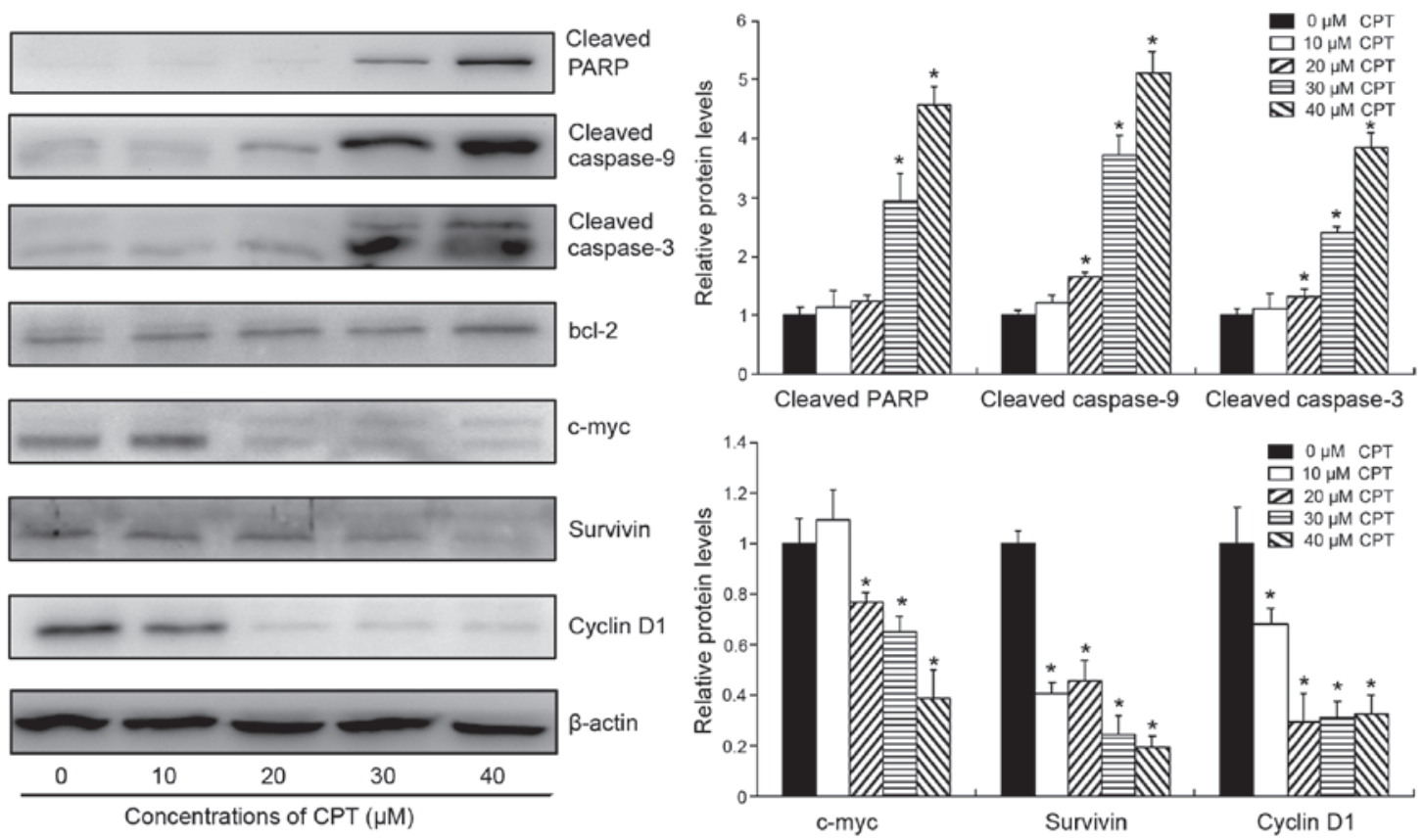

Figure 3. Western blot analyses of the apoptosis- and cell cycle-associated proteins in the BxPC-3 cells following CPT treatment. The BxPC-3 cells were exposed to different concentrations of CPT for $24 \mathrm{~h}$, following which the cells were subjected to western blot analysis to determine the expression levels of the indicated proteins. The density of the bands was further quantified using Gel-Pro Analyzer software. Data are expressed as the mean \pm standard deviation. ${ }^{*} \mathrm{P}<0.05$, vs. control group. CPT, cryptotanshinone; PARP, poly ADP ribose polymerase; bcl-2, B cell lymphoma-2.

by $10 \mu \mathrm{M} \mathrm{CPT}$, whereas the phosphorylation levels of the other upstream proteins were not altered or were increased only marginally by CPT at the same concentration. Furthermore, the inhibition of the upstream kinases appeared later than the inhibition of the STAT3 phosphorylation, according to the time course analysis (Fig. 4B). As shown in Fig. 4B, the suppression of STAT3 phosphorylation occurred with $30 \mathrm{~min}$ in the BxPC-3 cells. However, CPT had no effect on the activities of JAK2 or mTOR following $6 \mathrm{~h}$ treatment with $30 \mu \mathrm{M} \mathrm{CPT}$, and the phosphorylation level of Akt increased only marginally. In addition, the expression of p-ERK was decreased only when the treatment duration was increased to $2 \mathrm{~h}$. These results indicated that the inhibition of the STAT3 signaling pathway by CPT occurred through a mechanism independent of the above-mentioned upstream regulators, suggesting the possibility that CPT directly inhibited the phosphorylation of STAT3 in the BxPC-3 cells. The suppression of other signaling pathways following $24 \mathrm{~h}$ treatment appeared to be involved the secondary effects of CPT in human pancreatic cancer.

\section{Discussion}

In the present study, it was demonstrated that CPT inhibited the viability of BxPC-3 human pancreatic cancer cells by arresting cell cycle at the G1-G0 phase and inducing cell apoptosis. The anticancer effect of CPT is associated with inhibition of the activity of the STAT3 signaling pathway. The results of the present study demonstrated that CPT may be used a potential drug for adjuvant therapy or a complementary alternative medicine for the management of pancreatic cancer.

Despite significant advances in modern medicine during the last two decades, pancreatic cancer remains associated with a high rate of mortality (1). Cytotoxic chemotherapy based on the pyrimidine analogue, gemcitabine, remains the standard approach in the adjuvant and palliative setting, however, the resulting responses are minimal (19). Resistance to cytotoxic chemotherapy has remained a major factor contributing to the poor outcomes observed in patients with pancreatic cancer (20). The activation of STAT3 regulates oncogenic signaling in several types of tumor and leads to increased cell survival, proliferation and tumor growth (21). Activated STAT3 has been considered as a potential biomarker of chemotherapeutic resistance, and inhibition of the STAT3 pathway as a promising treatment strategy for pancreatic tumors (8). In the present study, the effect of CPT, a potent STAT3 pathway inhibitor, on BxPC-3 human pancreatic cells were investigated. The results revealed that $\mathrm{CPT}$ inhibited proliferation through the induction of apoptosis and cell cycle arrest in the BxPC-3 cells (Fig. 2). Furthermore, CPT decreased the activity of the STAT3 pathway in a dose- and time-dependent manner. Previous studies have indicated that CPT affects the STAT3 signaling pathway either directly or by alterations of certain upstream regulators $(14,22)$. The results of the present study demonstrated that CPT significantly inhibited the activities of JAK2, Akt, mTOR and ERK, when the duration of CPT exposure increased to $24 \mathrm{~h}$ (Fig. 3). However, CPT only affected the expression of p-STAT3 within $30 \mathrm{~min}$, and no significant effects on other proteins were observed, suggesting that $\mathrm{CPT}$ regulated the STAT3 signaling pathway directly in the BxPC-3 cells.

Phytochemicals from dietary plants and other plant sources, including herbs are becoming increasingly important sources of anticancer drugs or compounds for cancer chemopreventive or adjuvant chemotherapy (23). The use of traditional Chinese herbs has exhibit beneficial effects in overcoming apoptosis resistance in pancreatic cancer (24). CPT, a 
A
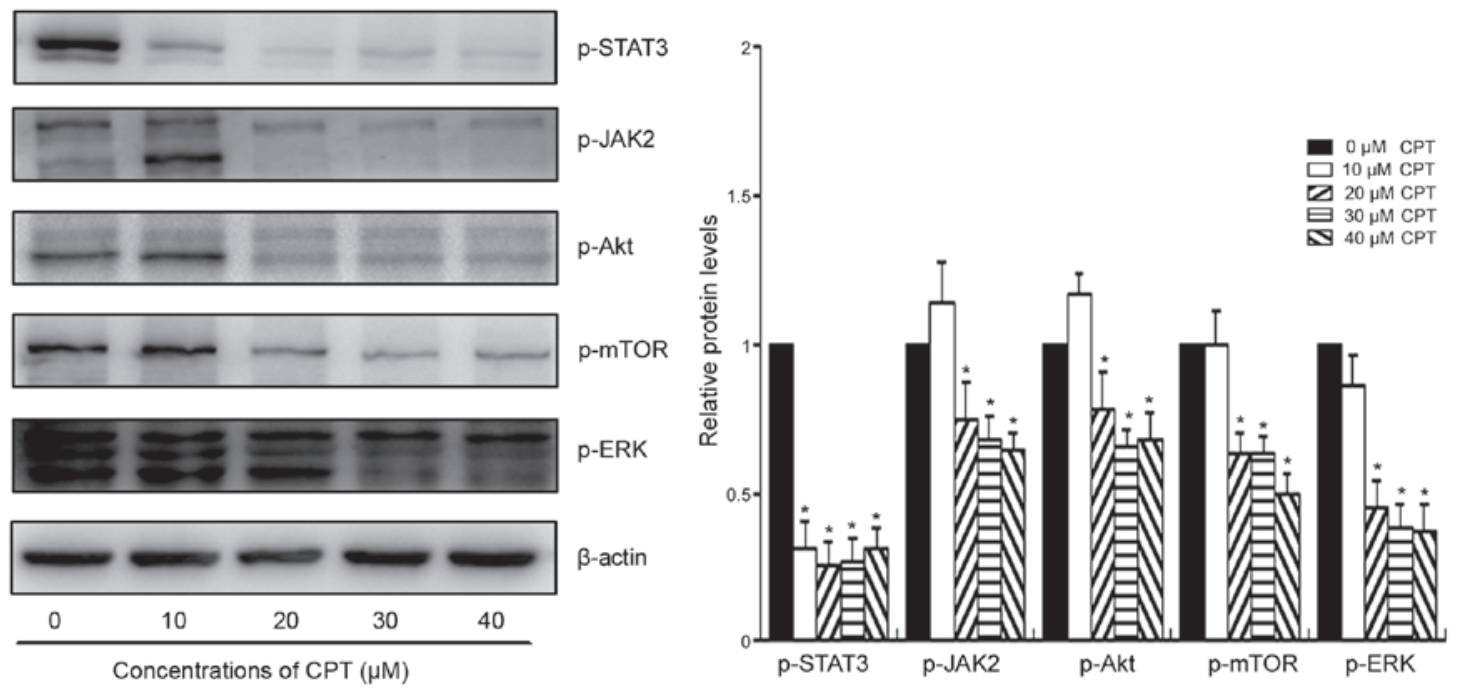

$\mathbf{B}$
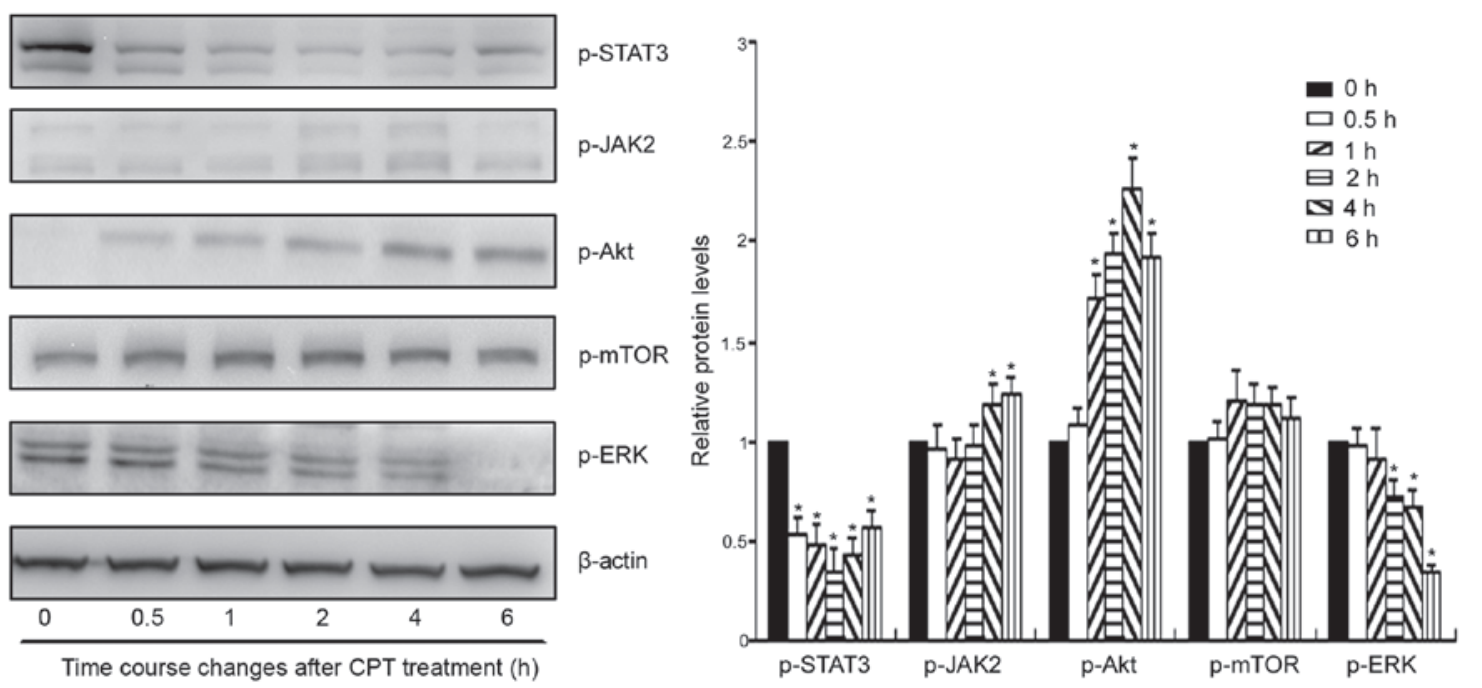

Figure 4. Effect of CPT on the activities of STAT3 and other survival-signaling pathways in the BxPC-3 cells. (A) Cells were treated with the indicated concentrations of CPT for $24 \mathrm{~h}$, following which the cells were subjected to western blot analysis to determine the expression levels of p-STAT3, p-JAK2, p-Akt, p-mTOR and p-ERK. (B) Time course analysis of the survival pathways following CPT treatment in the BxPC-3 cells. Cells were treated with $30 \mu \mathrm{M}$ CPT for the indicate durations, following which the cells were collected and subjected to western blot analysis. " $\mathrm{P}<0.05$, vs. control group $(0 \mathrm{~h})$. p-phosphorylated; STAT3, signal transducer and activator of transcription 3; JAK2, Janus kinase 2; mTOR, mammalian target of rapamycin; ERK, extracellular signal-regulated kinase.

biologically active compound from the root of Salvia miltiorrhiza, has exhibited significant anticancer effects in several tumor cells. The results from a study by Chen et al suggested that CPT is a potential drug for the treatment and prevention of human lung cancer (25). The results of our previous study indicated that CPT remained potent in tumor cells with an MDR phenotype, suggesting its potential application in cancer exhibiting a high level of chemotherapeutic resistance (12). In the present study, CPT significantly decreased the viability of the BxPC-3 pancreatic cancer cells via the induction of apoptosis and cell cycle arrest, suggesting the possibility of CPT being developed as an agent for pancreatic cancer therapy. Previous studies have indicated that compounds from herbal medicines may augment the sensitivity of pancreatic cancer cells to gemcitabine, and the combination therapy with natural products and cytotoxic agents has achieved promising results. Von Hoff et al demonstrated that the combination of nab-paclitaxel and gemcitabine significantly improve overall survival rates and response rates in patients with metastatic pancreatic adenocarcinoma (26). Enhanced antitumor efficacy has also been observed following treatment with emodin and gemcitabine in human pancreatic cancer cells $(27,28)$. Of note, CPT can also act synergistically with cytotoxic agents in other cancer cells (16). Thus, a combination therapeutic approach using CPT and gemcitabine in pancreatic cancer is promising and requires further investigations in the future.

In conclusion, the results of the present study provide the first evidence, to the best of our knowledge, that CPT inhibited the proliferation of BxPC-3 cells by inducing apoptosis and cell cycle arrest. The antitumor effect was found to be associated with inhibition of the STAT3 pathway. Taking into account the wide acceptance and low toxicity of plant extracts, the results of the present study suggest the possibility of further developing CPT as an alternative treatment option, or using it as adjuvant chemotherapeutic agent in the treatment of pancreatic cancer. 


\section{Acknowledgements}

This study was supported by the National Natural Science Foundation of China (grant. nos. 81202820 and 81303269), the Zhejiang Provincial Natural Science Foundation of China (grant. no. Q12H28005) and the Zhejiang Provincial Public Technology Applied Research Project (grant. no. 2014C33211).

\section{References}

1. Jemal A, Siegel R, Xu J and Ward E: Cancer statistics, 2010. CA Cancer J Clin 60: 277-300, 2010.

2. Wang BH, Cao WM, Yu J and Wang XL: Gemcitabine-based concurrent chemoradiotherapy versus chemotherapy alone in patients with locally advanced pancreatic cancer. Asian Pac J Cancer Prev 13: 2129-2132, 2012.

3. Matthaios D, Zarogoulidis P, Balgouranidou I, Chatzaki E and Kakolyris S: Molecular pathogenesis of pancreatic cancer and clinical perspectives. Oncology 81: 259-272, 2011.

4. Sehara Y, Sawicka K, Hwang JY, Latuszek-Barrantes A, Etgen AM and Zukin RS: Survivin is a transcriptional target of STAT3 critical to estradiol neuroprotection in global ischemia. J Neurosci 33: 12364-12374, 2013.

5. Yu H and Jove R: The STATs of cancer-new molecular targets come of age. Nat Rev Cancer 4: 97-105, 2004.

6. Takezawa K, Okamoto I, Nishio K, Jänne PA and Nakagawa K: Role of ERK-BIM and STAT3-survivin signaling pathways in ALK inhibitor-induced apoptosis in EML4-ALK-positive lung cancer. Clin Cancer Res 17: 2140-2148, 2011.

7. Lesina M, Kurkowski MU, Ludes K, Rose-John S, Treiber M, Klöppel G, Yoshimura A, Reindl W, Sipos B, Akira S, et al: Stat3/Socs 3 activation by IL-6 transsignaling promotes progression of pancreatic intraepithelial neoplasia and development of pancreatic cancer. Cancer Cell 19: 456-469, 2011.

8. Nagaraj NS, Washington MK and Merchant NB: Combined blockade of Src kinase and epidermal growth factor receptor with gemcitabine overcomes STAT3-mediated resistance of inhibition of pancreatic tumor growth. Clin Cancer Res 17: 483-493, 2011.

9. Li HD, Huang C, Huang KJ, Wu WD, Jiang T, Cao J, Feng ZZ and Qiu ZJ: STAT3 knockdown reduces pancreatic cancer cell invasiveness and matrix metalloproteinase-7 expression in nude mice. PLoS One 6: e25941, 2011.

10. Huang S and Sinicrope FA: Sorafenib inhibits STAT3 activation to enhance TRAIL-mediated apoptosis in human pancreatic cancer cells. Mol Cancer Ther 9: 742-750, 2010.

11. Park IJ, Kim MJ, Park OJ, Choe W, Kang I, Kim SS and Ha J: Cryptotanshinone induces ER stress-mediated apoptosis in HepG2 and MCF7 cells. Apoptosis 17: 248-257, 2012.

12. Ge Y, Cheng R, Zhou Y, Shen J, Peng L, Xu X, Dai Q, Liu P, Wang H, Ma X, et al: Cryptotanshinone induces cell cycle arrest and apoptosis of multidrug resistant human chronic myeloid leukemia cells by inhibiting the activity of eukaryotic initiation factor 4E. Mol Cell Biochem 368: 17-25, 2012.

13. Chen W, Luo Y, Liu L, Zhou H, Xu B, Han X, Shen T, Liu Z, Lu Y and Huang S: Cryptotanshinone inhibits cancer cell proliferation by suppressing Mammalian target of rapamycin-mediated cyclin D1 expression and Rb phosphorylation. Cancer Prev Res (Phila) 3: 1015-1025, 2010
14. Shin DS, Kim HN, Shin KD, Yoon YJ, Kim SJ, Han DC and Kwon BM: Cryptotanshinone inhibits constitutive signal transducer and activator of transcription 3 function through blocking the dimerization in DU145 prostate cancer cells. Cancer Res 69: 193-202, 2009.

15. Lu L, Li C, Li D, Wang Y, Zhou C, Shao W, Peng J, You Y, Zhang $\mathrm{X}$ and Shen X: Cryptotanshinone inhibits human glioma cell proliferation by suppressing STAT3 signaling. Mol Cell Biochem 381: 273-282, 2013

16. Ge Y, Yang B,Xu X,DaiQ,ChenZand Cheng R: Cryptotanshinone acts synergistically with imatinib to induce apoptosis of human chronic myeloid leukemia cells. Leuk Lymphoma 56: 730-738, 2015.

17. Papanikolaou V, Iliopoulos D, Dimou I, Dubos S, Kappas C, Kitsiou-Tzeli $\mathrm{S}$ and Tsezou A: Survivin regulation by HER2 through NF- $x \mathrm{~B}$ and c-myc in irradiated breast cancer cells. J Cell Mol Med 15: 1542-1550, 2011.

18. Sun M, Liu C, Nadiminty N, Lou W, Zhu Y, Yang J, Evans CP, Zhou Q and Gao AC: Inhibition of Stat 3 activation by sanguinarine suppresses prostate cancer cell growth and invasion. Prostate 72: 82-89, 2012.

19. Cao H, LE D and Yang LX: Current status in chemotherapy for advanced pancreatic adenocarcinoma. Anticancer Res 33: 1785-1791, 2013

20. Schüler S, Diersch S, Hamacher R, Schmid RM, Saur D and Schneider G: SKP2 confers resistance of pancreatic cancer cells towards TRAIL-induced apoptosis. Int J Oncol 38: 219-225, 2011.

21. Pandurangan AK and Esa NM: Signal transducer and activator of transcription 3-a promising target in colitis-associated cancer. Asian Pac J Cancer Prev 15: 551-560, 2014.

22. Don MJ, Liao JF, Lin LY and Chiou WF: Cryptotanshinone inhibits chemotactic migration in macrophages through negative regulation of the PI3K signaling pathway. Br J Pharmacol 151: 638-646, 2007

23. Ge YQ, Xu XF, Yang B, Chen Z and Cheng RB: Saponins from Rubus parvifolius L. induce apoptosis in human chronic myeloid leukemia cells through AMPK activation and STAT3 inhibition. Asian Pac J Cancer Prev 15: 5455-5461, 2014.

24. Zhang J, Wang P, Ouyang H, Yin J, Liu A, Ma C and Liu L: Targeting cancer-related inflammation: Chinese herbal medicine inhibits epithelial -to- mesenchymal transition in pancreatic cancer. PLoS One 8: e70334, 2013.

25. Chen L, Wang HJ, Xie W, Yao Y, Zhang YS and Wang H: Cryptotanshinone inhibits lung tumorigenesis and induces apoptosis in cancer cells in vitro and in vivo. Mol Med Rep 9: 2447-2452, 2014.

26. Von Hoff DD, Ervin T, Arena FP, Chiorean EG, Infante J, Moore M, Seay T, Tjulandin SA, Ma WW, Saleh MN, et al: Increased survival in pancreatic cancer with nab-paclitaxel plus gemcitabine. N Engl J Med 369: 1691-1703, 2013.

27. Wang ZH, Chen H, Guo HC, Tong HF, Liu JX, Wei WT, Tan W, Ni ZL, Liu HB and Lin SZ: Enhanced antitumor efficacy by the combination of emodin and gemcitabine against human pancreatic cancer cells via downregulation of the expression of XIAP in vitro and in vivo. Int J Oncol 39: 1123-1131, 2011.

28. Liu A, Chen H, Tong H, Ye S, Qiu M, Wang Z, Tan W, Liu J and Lin S: Emodin potentiates the antitumor effects of gemcitabine in pancreatic cancer cells via inhibition of nuclear factor- $\kappa \mathrm{B}$. Mol Med Rep 4: 221-227, 2011. 